\title{
Age-related differences in clinical characteristics of Kawasaki disease
}

\author{
Yu Peng ${ }^{1 * \oplus}$, Xiaohui Liu $^{1 * \oplus}$, Zhao Duan ${ }^{1 \oplus}$, Sufen Cai ${ }^{1 \oplus}$, Junkai Duan ${ }^{2 \oplus \bowtie}$, and Yulan Zhou ${ }^{3 \oplus \star}$ \\ ${ }^{1}$ Department of Rheumatology, Jiangxi Province Children's Hospital, Nanchang, Jiangxi, China \\ ${ }^{2}$ Department of Cardiology, Jiangxi Province Children's Hospital, Nanchang, Jiangxi, China \\ ${ }^{3}$ Department of Hematology, The First Affiliated Hospital of Nanchang University, Nanchang, Jiangxi, China
}

\begin{abstract}
This study aimed to examine and summarize clinical characteristics of Kawasaki disease (KD) at different ages to further strengthen clinicians understanding of children with KD, improving the level of diagnosis, and reducing coronary artery complications of KD. A total of 398 patients with KD who were diagnosed between January 2016 and December 2017 were reviewed retrospectively. These participants were allocated into three groups according to age: group $A(<1$ year, $n=62)$, group $B(\geqslant 1$ and $<5$ years, $n=286)$, and group $C(\geqslant 5$ years, $n=50)$. Clinical manifestations, laboratory results, and echocardiographic findings were compared among the groups. Most (71.86\%) patients with KD were aged 1-5 years. The prevalence of cervical lymphadenopathy was lowest in group A. The duration of fever before admission was longest in group A. The rate of cervical lymphadenopathy and laboratory data were different among the groups. Group A had higher frequencies of gastrointestinal involvement, neurological symptoms, and redness at the Bacillus Calmette-Guerin inoculation site than the other groups. Infants aged $<1$ year with KD often have a longer duration of fever before admission, a lower prevalence of cervical lymphadenopathy, and a higher prevalence of gastrointestinal and neurological symptoms.
\end{abstract}

Key words: Kawasaki disease; Infants; Coronary artery lesion; Clinical characteristics

\section{Introduction}

Kawasaki disease (KD) is an acute, self-limited vasculitis with a worldwide occurrence in children of different ethnic origins. Its prevalence is significantly higher in North-East Asian countries (notably Japan and China) than in nonAsian ethnicities like the United States $(1,2)$. Due to different meteorological factors, infectious agents, and improved economic conditions, previous studies have revealed changes in epidemiology of KD over time (3). The incidence rate increases yearly. Recently, several cases of Kawasakilike disease have been reported in various locations that coincide with the Sars-CoV-2 pandemic, making this disease one of the hot topics $(4,5)$. Most cases of KD occur between the ages of 1 and 5 years $(1,6)$, with fewer cases in children younger than 1 year or older than 5 years. These younger and older patients are more likely to have the incomplete type of KD. Therefore, diagnosis and treatment of KD could be delayed. Additionally, patients with delayed treatment have a higher risk of coronary artery lesions (CALs), which is the most serious complication of KD.

To increase clinicians understanding of children with $\mathrm{KD}$, improving the level of diagnosis, and reducing the coronary artery complications of $\mathrm{KD}$, we investigated the clinical characteristics of patients with KD at different ages to determine age-related differences in the course of this disease.

\section{Material and Methods}

\section{Patients}

We retrospectively reviewed the medical records of patients who had KD on admission at the Department of Rheumatology, Jiangxi Province Children's Hospital between January 2016 and December 2017. Patients who had insufficient clinical or laboratory data were excluded. Patients who fulfilled the diagnostic criteria of complete or incomplete KD according to the American Heart Association diagnostic guidelines (7) were enrolled in this study. The data were categorized into the following three groups by age at diagnosis: group $A(<1$ year, $n=62)$, group $B(\geqslant 1$ year and $<5$ years, $n=286)$, and group $C(\geqslant 5$ years, $n=50)$. The study was approved by the Medical Ethics Committee of Jiangxi Province

Correspondence: Junkai Duan: <yeduanjk@163.com > | Yulan Zhou: <wenxin_yl@163.com>

*These authors contributed equally to this work.

Received May 23, 2020 | Accepted October 9, 2020 
Children's Hospital (JXSETYY-YXKY-20180031). Written informed consent was not required because of the retrospective nature of the study, and all identifying information of the enrolled patients was protected.

\section{Definitions}

CALs were defined as an internal lumen diameter $\geqslant 3 \mathrm{~mm}$ in children aged $<5$ years or $\geqslant 4 \mathrm{~mm}$ in children aged $\geqslant 5$ years (8). Intravenous immunoglobulin (IVIG) resistance was defined as recurrent or persistent fever for at least 36 hours after the end of IVIG administration (7). Gastrointestinal disorders included abdominal pain, hepatitis, diarrhea, vomiting, jaundice, gallbladder hydrops, and pancreatitis. Respiratory disorders included peribronchial and interstitial infiltrates on chest radiography. Genitourinary disorders included urethritis, hydrocele, and phimosis. Neurological disorders included aseptic meningitis, extreme irritability, and transient peripheral facial nerve palsy. Musculoskeletal disorders included arthralgia and arthritis. Redness at the Bacillus Calmette-Guerin (BCG) inoculation site, which was recorded precisely before and during hospitalization, was defined as any redness, induration, or crust formation.

\section{Data collection}

Data on demographic, clinical, laboratory, and echocardiographic characteristics were recorded. Demographic and clinical characteristics included age, sex, KD-associated manifestations, and clinical outcomes. Laboratory tests were performed on the first day of hospitalization. Two-dimensional echocardiography was used to assess cardiovascular complications, and this was performed at the time of diagnosis, several times a week during hospitalization, and 2 months after diagnosis.

\section{Statistical analysis}

Numerical data are reported as means \pm SD or median with interquartile range. Categorical variables are reported as numbers and percentages. We used one-way analysis of variance to analyze differences between groups based on numerical variables that showed a normal distribution. For analysis of variables not showing a normal distribution, we used the Kruskal-Wallis test. For analysis of categorical variables, we used the chi-squared test or Fisher's exact test. For variables that were significantly different among the three groups, pairwise comparisons were performed using the Bonferroni post hoc test. Statistical analyses were performed using SPSS version 22.0 for Windows (IBM, USA). A two-sided $\mathrm{P}<0.05$ was considered to be statistically significant.

\section{Results}

\section{Clinical characteristics among patients with KD with different ages}

Three hundred ninety-eight patients with KD with an age range of 2 months to 12 years were enrolled in this study. Baseline characteristics are summarized in Table 1. There was a significant difference in age at diagnosis of KD among the three groups $(P<0.001)$. There were $258(64.82 \%)$ males in this cohort, with no significant difference in sex distribution among the different age groups. The prevalence of incomplete type of KD was significantly different among the groups $(P<0.001)$. Group $B$ had a relatively lower proportion of the incomplete type than group A, but there was no significant difference between groups $A$ and $C$ and groups $B$ and $C$. The duration of fever before admission was significantly longer in group $A$ than in the other age groups $(P=0.034)$. Except

Table 1. Clinical parameters in the different Kawasaki disease (KD) age groups.

\begin{tabular}{|c|c|c|c|c|c|}
\hline Variable & Group A & Group B & Group C & F or $\chi^{2}$ value & $P$ value \\
\hline Number of patients & 62 & 286 & 50 & & \\
\hline Age at KD diagnosis (months) & $8.48 \pm 1.12$ & $24.15 \pm 14.67^{*}$ & $86.82 \pm 23.75^{\star, \star \star}$ & 173.67 & $<0.001^{\#}$ \\
\hline Male, n (\%) & $40(64.5 \%)$ & $186(65.0 \%)$ & $32(64.0 \%)$ & 0.023 & 0.989 \\
\hline Incomplete KD, n (\%) & $28(45.2 \%)$ & $66(23.1 \%)^{*}$ & $18(36.0 \%)$ & 14.036 & $<0.001^{\#}$ \\
\hline Fever duration, days (mean $\pm S D$ ) & $7.4 \pm 2.4$ & $6.9 \pm 1.9^{*}$ & $7.1 \pm 2.1^{*}$ & 6.235 & $0.034^{\#}$ \\
\hline Rash, n (\%) & $34(54.8 \%)$ & $184(64.3 \%)$ & $26(52.0 \%)$ & 4.025 & 0.134 \\
\hline Cervical lymphadenopathy, n (\%) & $18(29.0 \%)$ & $183(64.0 \%)^{*}$ & $32(64.0 \%)^{*}$ & 26.352 & $<0.001^{\#}$ \\
\hline Conjunctivitis, n (\%) & $56(90.3 \%)$ & $242(84.6 \%)$ & $41(82.0 \%)$ & 1.771 & 0.412 \\
\hline Oral changes, n (\%) & $54(87.1 \%)$ & $234(81.8 \%)$ & $38(76.0 \%)$ & 2.306 & 0.316 \\
\hline Extremity changes, $\mathrm{n}(\%)$ & $22(35.5 \%)$ & $130(45.5 \%)$ & $16(32.0 \%)$ & 4.521 & 0.104 \\
\hline IVIG-resistant, n (\%) & $15(24.2 \%)$ & $52(18.2 \%)$ & $10(20.0 \%)$ & 1.196 & 0.550 \\
\hline
\end{tabular}

Group $\mathrm{A}:<1$ year; group $\mathrm{B}: \geqslant 1$ and $<5$ years; group $\mathrm{C}: \geqslant 5$ years; IVIG: intravenous immunoglobulin. ${ }^{\text {\# }}<0.05$ among Groups $\mathrm{A}, \mathrm{B}$, and C; ${ }^{*} \mathrm{P}<0.05$ compared with Group A; ${ }^{* *} \mathrm{P}<0.05$ compared with Group B. The chi-squared test, Fisher's (F) exact test, or one-way analysis of variance were used for statistical analyses. 
Table 2. Other clinical findings in the different Kawasaki disease age groups.

\begin{tabular}{|c|c|c|c|c|c|}
\hline Variable & Group A & Group B & Group C & $\chi^{2}$ value & $P$ value \\
\hline Gastrointestinal, n (\%) & $16(25.8 \%)$ & $30(10.5 \%)^{*}$ & $9(18.0 \%)$ & 10.877 & $0.004^{\#}$ \\
\hline Abdominal pain, $\mathrm{n}(\%)$ & $0(0 \%)$ & $15(5.2 \%)$ & $3(6 \%)$ & 3.941 & 0.147 \\
\hline Hepatitis, n (\%) & $0(0 \%)$ & $0(0 \%)$ & $1(2 \%)$ & 4.742 & 0.126 \\
\hline Diarrhea, n (\%) & $10(16.1 \%)$ & $13(4.5 \%)^{\star}$ & $2(4 \%)$ & 9.635 & $0.006^{\#}$ \\
\hline Vomiting, n (\%) & $8(12.9 \%)$ & $10(3.5 \%)^{*}$ & $1(2 \%)$ & 8.430 & $0.011^{\#}$ \\
\hline Jaundice, n (\%) & $2(3.2 \%)$ & $1(0.3 \%)$ & $1(2 \%)$ & 5.116 & 0.069 \\
\hline Gallbladder hydrops, n (\%) & $1(1.6 \%)$ & $2(0.7 \%)$ & $2(4 \%)$ & 3.928 & 0.091 \\
\hline Pancreatitis, n (\%) & $0(0 \%)$ & $2(0.7 \%)$ & $1(2 \%)$ & 1.672 & 0.338 \\
\hline Respiratory, n (\%) & $8(12.9 \%)$ & $54(18.9 \%)$ & $7(14.0 \%)$ & 1.715 & 0.424 \\
\hline Genitourinary, n (\%) & $7(11.3 \%)$ & $21(7.1 \%)$ & $5(10.0 \%)$ & 1.264 & 0.532 \\
\hline Neurological, n (\%) & $12(19.4 \%)$ & $18(6.3 \%)^{*}$ & $2(4.0 \%)^{\star}$ & 10.525 & $0.003^{\#}$ \\
\hline Aseptic meningitis, $\mathrm{n}(\%)$ & $5(8.1 \%)$ & $14(4.9 \%)$ & $2(4 \%)$ & 1.279 & 0.550 \\
\hline Extreme irritability, n (\%) & $10(16.1 \%)$ & $9(3.1 \%)^{*}$ & $0(0 \%)^{*}$ & 15.978 & $<0.001^{\#}$ \\
\hline Transient peripheral facial nerve palsy, $\mathrm{n}(\%)$ & $0(0 \%)$ & $3(1 \%)$ & $0(0 \%)$ & 0.395 & 1.000 \\
\hline Musculoskeletal, n (\%) & $1(1.6 \%)$ & $35(12.2 \%)^{*}$ & $7(14.0 \%)^{*}$ & 6.309 & $0.043^{\#}$ \\
\hline Redness at the BCG inoculation site, $\mathrm{n}(\%)$ & $27(43.5 \%)$ & $18(6.3 \%)^{*}$ & $0(0.0 \%)^{\star}$ & 77.811 & $<0.001^{\#}$ \\
\hline
\end{tabular}

Group A: $<1$ year; group B: $\geqslant 1$ and $<5$ years; group C: $\geqslant 5$ years. ${ }^{\#} \mathrm{P}<0.05$ among Groups $\mathrm{A}$, B, and C; ${ }^{*} \mathrm{P}<0.05$ compared with Group A. The chi-squared test was used for statistical analyses.

for fever, the most frequent symptom was bulbar conjunctival injection (339 cases, $85.2 \%$ ), followed by oral changes (326 cases, $81.9 \%$ ), rash (244 cases, $61.3 \%)$, cervical lymphadenopathy (239 cases, 60.1\%), and changes in the extremities (168 cases, 42.2\%). The frequency of cervical lymphadenopathy was lowest in group $A$, with a significant difference among the groups $(P<0.001)$. There were no significant differences in the frequency of rash, bulbar conjunctival injection, oral changes, and changes in the extremities among the different age groups.

Except for the classic clinical features, multiple other organs and tissues were inflamed during the acute illness and caused clinical symptoms. We analyzed these clinical symptoms in the different age groups. As shown in Table 2, $55(13.8 \%)$ patients had gastrointestinal involvement, $69(17.3 \%)$ had respiratory involvement, $33(8.3 \%)$ had genitourinary involvement, $32(8.0 \%)$ had neurological involvement, $43(10.8 \%)$ had musculoskeletal involvement, and $45(11.3 \%)$ had redness at the BCG inoculation site. There was a significant difference in gastrointestinal involvement among the groups $(P=0.004)$, with group $A$ having a higher frequency than group $B$, but with no significant difference between groups $A$ and $C$ and between groups $\mathrm{B}$ and $\mathrm{C}$. The rates of neurological involvement and redness at the BCG inoculation site were significantly higher in group $A$ than in groups $B$ and $C$ $(P=0.003$ and $P<0.001$, respectively). However, the frequency of musculoskeletal disorders in group $A$ was significantly lower than that in groups $B$ and $C(P=0.043)$. There were no significant differences in the frequencies of respiratory disorders and genitourinary disorders among the different age groups. We further analyzed the number of patients with each gastrointestinal or neurological presentation in the different age groups. We found that the frequency of diarrhea and vomiting in group $A$ was significantly higher than that in group $B(P=0.006$ and $P=0.011$, respectively), but there were no significant differences between groups $A$ and $C$ or between groups $B$ and $C$. The frequency of extreme irritability was highest in group $A(P<0.001)$. There were no differences in the frequencies of other gastrointestinal or neurological presentations among the different age groups.

\section{Laboratory findings among patients with KD with different ages}

We then compared laboratory data, including white blood cell count, neutrophils, hemoglobin, platelet count, C-reactive protein levels, erythrocyte sedimentation rate, and the levels of procalcitonin, serum sodium, lactate dehydrogenase, alanine aminotransferase, aspartate aminotransferase, and brain natriuretic peptide, in patients with $\mathrm{KD}$ according to age. However, there were no significant differences in these laboratory findings among the different age groups (Table 3).

\section{Echocardiographic findings among patients with KD with different ages}

Initial echocardiographic findings indicated that there was no significant difference in the incidence of CALs among the different age groups. However, the incidence of CALs in group A was significantly higher than that in the other groups 2 months after diagnosis ( $P=0.036$, Table 4). Other echocardiographic findings, including pericardial effusion (16 cases, 4.0\%), decreased left ventricular function (9 cases, $2.3 \%$ ), mitral regurgitation (6 cases, 
Table 3. Laboratory data of the different Kawasaki disease age groups.

\begin{tabular}{lcccc}
\hline Variable & Group A & Group B & Group C & P value \\
\hline WBC $\left(10^{9} / \mathrm{L}\right)$ & $17.31 \pm 6.31$ & $16.08 \pm 5.65$ & $16.03 \pm 4.32$ & 3.034 \\
N $\left(10^{9} / \mathrm{L}\right)$ & $10.36 \pm 4.47$ & $10.94 \pm 4.94$ & $10.79 \pm 4.51$ & 0.087 \\
Hb $(\mathrm{g} / \mathrm{L})$ & $106.28 \pm 12.72$ & $103.75 \pm 10.89$ & $109.29 \pm 7.39$ & 0.151 \\
PLT $\left(10^{9} / \mathrm{L}\right)$ & $395.24 \pm 141.33$ & $357.08 \pm 111.38$ & $376.00 \pm 78.25$ & 0.860 \\
CRP $(\mathrm{mg} / \mathrm{L})$ & $90.42 \pm 58.25$ & $103.80 \pm 66.85$ & $112.44 \pm 60.13$ & 0.710 \\
ESR $(\mathrm{mm} / \mathrm{h})$ & $66.44 \pm 25.34$ & $63.37 \pm 26.63$ & $65.35 \pm 25.07$ & 0.654 \\
PCT $(\mu \mathrm{g} / \mathrm{L})$ & $10.38 \pm 43.96$ & $4.93 \pm 40.44$ & $1.23 \pm 2.30$ & 0.588 \\
Na $(\mathrm{mmol} / \mathrm{L})$ & $138.00 \pm 2.41$ & $137.30 \pm 3.07$ & $136.18 \pm 3.24$ & 0.310 \\
LDH $(\mathrm{U} / \mathrm{L})$ & $405.92 \pm 125.03$ & $449.08 \pm 197.85$ & $443.94 \pm 211.52$ & 0.611 \\
ALT $[\mathrm{U} / \mathrm{L}]$ & $28(20-60)$ & $38(19-125)$ & $27(18-73)$ & 0.734 \\
AST $[\mathrm{U} / \mathrm{L}]$ & $38.5(29-56)$ & $41(31-74)$ & $31(23-70)$ & 0.158 \\
BNP $[\mathrm{U} / \mathrm{L}]$ & $513(269-1058)$ & $332(183-819)$ & $308(158-545)$ & 0.585 \\
\hline
\end{tabular}

Group A: $<1$ year; group B: $\geqslant 1$ and $<5$ years; group C: $\geqslant 5$ years. WBC: white blood cell; N: neutrophils; Hb: hemoglobin; PLT: platelet count; CRP: C-reactive protein; ESR: erythrocyte sedimentation rate; PCT: procalcitonin; Na: serum sodium; LDH: lactate dehydrogenase; ALT: alanine aminotransferase; AST aspartate aminotransferase; BNP: brain natriuretic peptide. Data are reported as means \pm SD or median with interquartile range. The chi-squared test, Fisher's $(F)$ exact test, or one-way analysis of variance were used for statistical analyses.

Table 4. Echocardiographic findings in the different Kawasaki disease age groups.

\begin{tabular}{|c|c|c|c|c|c|}
\hline Variable & Group A & Group B & Group C & $\chi^{2}$ value & $P$ value \\
\hline Initial CAL, n (\%) & $15(24.2 \%)$ & $45(15.7 \%)$ & $8(16.0 \%)$ & 2.621 & 0.270 \\
\hline CAL at 2 months follow-up, n (\%) & $12(19.4 \%)$ & $24(8.4 \%)^{\star}$ & $5(10.0 \%)^{*}$ & 6.633 & $0.036^{\#}$ \\
\hline Pericardial effusion, n (\%) & $4(6.5 \%)$ & $9(3.1 \%)$ & $3(6.0 \%)$ & 2.557 & 0.293 \\
\hline Decreased left ventricular systolic function, $\mathrm{n}(\%)$ & $1(1.6 \%)$ & $6(2.1 \%)$ & $2(4.0 \%)$ & 1.127 & 0.651 \\
\hline Mitral regurgitation, n (\%) & $3(4.8 \%)$ & $3(1 \%)$ & $0(0 \%)$ & 4.226 & $0.097^{\#}$ \\
\hline Aortic regurgitation, $\mathrm{n}(\%)$ & $2(3.2 \%)$ & $6(2.1 \%)$ & $2(4 \%)$ & 1.407 & 0.516 \\
\hline
\end{tabular}

Group $A$ : $<1$ year; group $B: \geqslant 1$ and $<5$ years; group $C: \geqslant 5$ years. CAL: coronary artery lesions. ${ }^{\#} P<0.05$ among Groups $A, B$, and $C$; ${ }^{*} \mathrm{P}<0.05$ compared with Group A. The chi-squared test was used for statistical analyses.

$1.5 \%$ ), and aortic regurgitation (10 cases, $2.5 \%$ ), were not significantly different among the age groups.

\section{Discussion}

In our study, the male/female ratio was 1.84 , similar to those from several national epidemiological surveys on KD (9-11). However, there was no obvious difference in sex distribution among the different age groups.

Previous studies have suggested that KD patients with different ages have different manifestations of the disease $(12,13)$. In our study, the prevalence of symptoms of oral changes, conjunctivitis, rash, and changes in the extremities was not significantly different among the different age groups. However, the prevalence of cervical lymphadenopathy was lower in patients aged $<1$ year than in those at other ages, and these patients were more likely to have incomplete clinical presentation. These results showed similarities with earlier results obtained by Cho et al. (14), who reported that the least common symptom in patients with KD aged $<1$ year was cervical lymphadenopathy. We also found that the duration of fever before admission was significantly longer in patients aged $<1$ year than in the other age groups, which presumably was attributed to the high proportion of incomplete KD in these patients.

Gastrointestinal disorders include many different clinical manifestations and echographic findings, which have been reported in approximately 20 to $35 \%$ of patients with KD (15-18). In a recent study, Fabiet al. (17) found that patients with KD and gastrointestinal involvement were significantly younger. Our findings were similar as gastrointestinal disorders occurred more frequently in patients aged $<1$ year than in those aged $1-5$ years. Neurological involvement is a relatively unrecognized complication of $\mathrm{KD}$, and it includes extreme irritability (19), aseptic meningitis (20), peripheral facial nerve palsy (21), and sensorineural hearing loss (22). However, neurological involvement has been reported mainly in case reports and is poorly characterized. In our cohort, the proportion of 
infants aged $<1$ year with neurological involvement was significantly higher than that of older patients.

Redness at the BCG inoculation site was described as a positive sign in the American Heart Association scientific statement (7). In a Japanese nationwide epidemiological survey, Uehara et al. (23) reported that $49.9 \%$ of patients with $\mathrm{KD}$ and a history of BCG vaccination had redness at the BCG inoculation site. Moreover, of those patients aged 3-20 months, more than $70 \%$ had redness at the BCG inoculation site. In this study, young patients were also more prone to developing redness at the BCG inoculation site. Furthermore, redness at the BCG inoculation site was more prevalent than cervical lymphadenopathy and changes in the extremities in patients with $\mathrm{KD}$ aged $<1$ year. Redness at the BCG inoculation site appears to be a useful criterion in the diagnosis of KD in younger infants.

Musculoskeletal involvement includes arthralgia and arthritis. Previous studies showed that the prevalence of arthritis in KD ranged from 7.5 to $12.7 \%$ (24), and older children with KD were more prone to developing arthritis. Similar results were found in our study, where the frequency of musculoskeletal involvement in patients aged $<1$ year was lower than that in older patients. We speculate that this finding was mainly due to the difficulty of diagnosing arthritis in this age group.

Respiratory symptoms are frequently observed in children with KD during the acute phase (25). Lee et al. (26) reported that respiratory symptoms were found in $31.9 \%$ of patients with $\mathrm{KD}$, and there was no significant difference in age distribution between patients with KD and without respiratory symptoms. Genitourinary involvement of KD is uncommon and most patients with KD and genitourinary involvement have pyuria (7). Watanabe (27) reported that patients with $K D$ and pyuria were more likely to be aged $<1$ year. In our study, no significant difference was found in the prevalence of respiratory involvement or genitourinary involvement among the different age groups.

\section{References}

1. Kim GB. Reality of Kawasaki disease epidemiology. Korean J Pediatr 2019; 62: 292-296, doi: 10.3345/kjp. 2019.00157.

2. Hedrich $\mathrm{CM}$, Schnabel A, Hospach T. Kawasaki disease. Front Pediatr 2018; 6: 198, doi: 10.3389/fped.2018.00198.

3. Sun L, Tang Y, Wang Y, Qian G, Yan W, Wang B, et al. Cardiology 2018; 141: 25-31, doi: 10.1159/000493420.

4. Pouletty M, Borocco C, Ouldali N, Caseris M, Basmaci R, Lachaume $\mathrm{N}$, et al. Paediatric multisystem inflammatory syndrome temporally associated with SARS-CoV-2 mimicking Kawasaki disease (Kawa-COVID-19): a multicentre cohort. Ann Rheum Dis 2020; 79: 999-1006, doi: 10.1136/ annrheumdis-2020-217960.
Cardiovascular manifestations represent the major contributors to morbidity and mortality related to KD. Several studies have reported that young age is a risk factor for CALs $(28,29)$. Fernandez-Cooke et al. (11) studied the epidemiology and clinical features in KD in Spain and found that children aged $<12$ months develop coronary aneurysms more frequently than older children. In this study, we found that the rate of CALs in the acute phase of KD in patients aged <1year was not significantly different than that in older patients. However, there was a significantly higher incidence of CALs in younger patients at 2 months after initial echocardiography compared with older patients, which was similar to previous studies.

There are several limitations in this study. First, there was a limited number of patients enrolled in groups $A$ and $\mathrm{C}$, and thus more studies are required to investigate the specific characteristics of these patients. Second, detection of symptoms before hospitalization was partly based on interviews with the parents, who may have failed to recognize some symptoms.

\section{Conclusions}

Although other authors had a similar approach, our study added to the current knowledge on KD. Our study showed that cervical lymphadenopathy and musculoskeletal symptoms occurred more frequently in older patients than in younger patients. Gastrointestinal symptoms, neurological symptoms, and redness at the BCG inoculation site occurred more frequently in patients aged $<1$ year than in older patients, and these patients were more likely to develop CALs.

\section{Acknowledgments}

The authors would like to acknowledge the support of the Science Program of Jiangxi Administration of Traditional Chinese Medicine (No. 2019A214) and the Science Program of Health Commission of Jiangxi Province (No. 20203111).

5. Jones VG, Mills M, Suarez D, Hogan CA, Yeh D, Segal JB, et al. COVID-19 and Kawasaki disease: novel virus and novel case. Hosp Pediatr 2020; 10: 537-540, doi: 10.1542/ hpeds.2020-0123.

6. Uehara R, Belay ED. Epidemiology of Kawasaki Disease in Asia, Europe, and the United States. J Epidemiol 2012; 22, 79-85, doi: 10.2188/jea.JE20110131.

7. McCrindle BW, Rowley AH, Newburger JW, Burns JC, Bolger AF, Gewitz M, et al. Diagnosis, treatment, and longterm management of Kawasaki disease: a scientific statement for health professionals from the American Heart Association. Circulation 2017; 135: e927-e999, doi: 10.1161/CIR.000000 0000000484 
8. JCS Joint Working Group. Guidelines for diagnosis and management of cardiovascular sequelae in Kawasaki disease (JCS 2013). Digest version. Circ J 2014; 78: $2521-$ 2562, doi: 10.1253/circj.CJ-66-0096.

9. Makino N, Nakamura Y, Yashiro M, Kosami K, Matsubara Y, Ae R, et al. Nationwide epidemiologic survey of Kawasaki disease in Japan, 2015-2016. Pediatr Int 2019; 61: 397403, doi: 10.1111/ped.13809.

10. Kim GB, Park S, Eun LY, Han JW, Lee SY, Yoon KL, et al. Epidemiology and clinical features of Kawasaki disease in South Korea, 2012-2014. Pediatr Infect Dis J 2017; 36: 482485, doi: 10.1097/INF.0000000000001474.

11. Fernandez-Cooke E, Barrios Tascon A, Sanchez-Manubens J, Anton J, Grasa Lozano CD, Aracil Santos J, Villalobos Pinto E, et al. Epidemiological and clinical features of Kawasaki disease in Spain over 5 years and risk factors for aneurysm development. (2011-2016): KAWA-RACE study group. PLoS One 2019; 14: e0215665, doi: 10.1371/journal. pone.0215665.

12. Shiozawa $Y$, Inuzuka $R$, Harita $Y$, Kagawa J. Age-related differences in the course of the acute phase symptoms of Kawasaki disease. Pediatr Infect Dis J 2013; 32: e365-369, doi: 10.1097/INF.0b013e3182952027.

13. Yoon YM, Yun HW, Kim SH. Clinical characteristics of Kawasaki disease in infants younger than six months: a single-center study. Korean Circ J 2016; 46: 550-555, doi: 10.4070/kcj.2016.46.4.550.

14. Cho MA, Choi YJ, Jung JW. Effects of "age at diagnosis" on coronary artery lesions in patients with incomplete Kawasaki disease. Korean Circ J 2010; 40: 283-287, doi: 10.4070/ kcj.2010.40.6.283.

15. Chen CJ, Huang FC, Tiao MM, Huang YH, Lin LY, Yu HR, et al. Sonographic gallbladder abnormality is associated with intravenous immunoglobulin resistance in Kawasaki disease. Scientific World J 2012; 2012: 485758, doi: 10.1100/ 2012/485758

16. Yi DY, Kim JY, Choi EY, Choi JY, Yang HR. Hepatobiliary risk factors for clinical outcome of Kawasaki disease in children. BMC Pediatr 2014; 14: 51, doi: 10.1186/14712431-14-51.

17. Fabi M, Corinaldesi E, Pieranton L, Mazzoni E, Landini C, Bigucci B, et al. Gastrointestinal presentation of Kawasaki disease: a red flag for severe disease? PLoS One 2018; 13 : e0202658, doi: 10.1371/journal.pone.0202658.
18. Yaniv L, Jaffe M, Shaou R. The surgical manifestations of the intestinal tract in Kawasaki disease. J Pediatr Surg 2005; 40: e1-e4, doi: 10.1016/j.jpedsurg.2005.05.063.

19. Thapa R, Chakrabartty S. Atypical Kawasaki disease with remarkable paucity of signs and symptoms. Rheumatol Int 2009; 29: 1095-1096, doi: 10.1007/s00296-009-0899-2.

20. Rossi M, Siani P, Grossi A, Carannante N, Di Caprio G, Borrelli B, et al. Aseptic meningitis as onset of Kawasaki disease. Minerva Pediatr 2020; 72: 135-137, doi: 10.23736/ S0026-4946.19.05384-2.

21. Yuan $\mathrm{Y}, \mathrm{Lu} \mathrm{N}$. Facial nerve palsy presenting as rare neurological complication of Kawasaki disease: a case report. Medicine (Baltimore) 2019; 98: e16888, doi: 10.1097/ md.0000000000016888.

22. Aggarwal V, Etinger V, Orjuela AF.Sensorineural hearing loss in Kawasaki disease. Ann Pediatr Cardiol 2016; 9: 8789, doi: 10.4103/0974-2069.171396.

23. Uehara $R$, Igarashi $H$, Yashiro $M$, Nakamura $Y$, Yanagawa $H$. Kawasaki disease patients with redness or crust formation at the Bacille Calmette-Guérin inoculation site. Pediatr Infect Dis J 2010; 29: 430-433, doi: 10.1097/INF.0b013e3181cacede.

24. Peng Y, Liu X, Duan Z, Deng Y, Cai S, Wang Z, et al. Prevalence and characteristics of arthritis in Kawasaki disease: a Chinese cohort study. Clin Exp Med 2019; 19: 167-172, doi: 10.1007/s10238-019-00547-w.

25. Chang LY, Lu CY, Shao PL, Lee PI, Lin MT, Fan TY, et al. J Formos Med Assoc 2014; 113; 148-154, doi: 10.1016/ j.jfma.2013.12.008.

26. Lee SB, Choi HS, Son S, Hong YM. Cardiac function in Kawasaki disease patients with respiratory symptoms. Korean Circ J 2015; 45: 317-324, doi: 10.4070/kcj.2015. 45.4.317.

27. Watanabe T. Pyuria in patients with Kawasaki disease. World J Clin Pediatr 2015: 4: 25-29, doi: 10.5409/wjcp.v4.i2.25.

28. Flores-Montes OA, Valle-Leal J, Arreguin-Reyes R, ArmentaVelderrain JM. Risk factors related to cardiovascular complications in children diagnosed with Kawasaki disease in Northwestern Mexico. Bol Med Hosp Infant Mex 2018; 75: 123-1129, doi: 10.24875/BMHIME.M18000033.

29. Son MBF, Gauvreau K, Tremoulet AH, Lo M, Baker AL, de Ferranti S, et al. Risk model development and validation for prediction of coronary artery aneurysms in Kawasaki disease in a North American population. J Am Heart Assoc 2019; 8: e011319, doi: 10.1161/JAHA.118.011319. 